This item was submitted to Loughborough's Research Repository by the author.

Items in Figshare are protected by copyright, with all rights reserved, unless otherwise indicated.

\title{
Presence and duration of reactivity to pedometers in adults
}

PLEASE CITE THE PUBLISHED VERSION

http://dx.doi.org/10.1249/MSS.0b013e318242a377

\section{PUBLISHER}

(c) American College of Sports Medicine

\section{VERSION}

AM (Accepted Manuscript)

\section{LICENCE}

CC BY-NC-ND 4.0

\section{REPOSITORY RECORD}

Clemes, Stacy A., and Nuala K. Deans. 2019. "Presence and Duration of Reactivity to Pedometers in Adults". figshare. https://hdl.handle.net/2134/15570. 
This item was submitted to Loughborough's Institutional Repository (https://dspace.lboro.ac.uk/) by the author and is made available under the following Creative Commons Licence conditions.

\section{creative
commons}

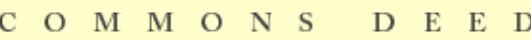

Attribution-NonCommercial-NoDerivs 2.5

You are free:

- to copy, distribute, display, and perform the work

Under the following conditions:

Attribution. You must attribute the work in the manner specified b the author or licensor.

Noncommercial. You may not use this work for commercial purposes.

No Derivative Works. You may not alter, transform, or build upon this work.

- For any reuse or distribution, you must make clear to others the license terms of this work.

- Any of these conditions can be waived if you get permission from the copyright holder.

Your fair use and other rights are in no way affected by the above.

This is a human-readable summary of the Leqal Code (the full license).

\section{Disclaimer 만}

For the full text of this licence, please go to: http://creativecommons.org/licenses/by-nc-nd/2.5/ 
The presence and duration of reactivity to pedometers in adults

Stacy A Clemes and Nuala K Deans

\section{Institutional affiliation of both authors:}

School of Sport, Exercise and Health Sciences, Loughborough University

\section{Corresponding Author:}

Dr Stacy A Clemes

School of Sport, Exercise and Health Sciences

Loughborough University

Loughborough

Leicestershire

LE11 3TU, UK

Telephone: $\quad+441509228170$

Fax: $\quad+441509223940$

Email: $\quad$ S.A.Clemes@lboro.ac.uk

Running title: Reactivity to pedometers in adults

Conflicts of Interest and Source of Funding: There are no perceived conflicts of interest associated with this research. No external funding supported the work outlined in the manuscript, the study was conducted using resources already available within the School of Sport, Exercise and Health Sciences. 


\section{ABSTRACT}

Purpose: To examine the presence and duration of reactivity to wearing a pedometer and recording daily step counts in free-living adults.

Methods: On the first visit to the laboratory 90 participants (69\% female, age $=26.8 \pm 13.0$ years, BMI $=23.4 \pm 4.0 \mathrm{~kg} / \mathrm{m}^{2}$ ), blinded to the study aim, were provided with a sealed pedometer (New Lifestyles NL-800) and informed that it was a 'Body Posture Monitor' (covert condition). Participants wore the pedometer throughout waking hours for 1 week. Upon return to the laboratory, stored step counts were recorded and participants were informed that the device was a pedometer. Participants wore the pedometer unsealed (no restriction on viewing the step count display) for 2 weeks, during which they recorded their daily step count in a diary (diary condition). Mean daily step counts recorded during the covert condition and during weeks 1 and 2 of the diary condition were compared using a repeated-measures ANOVA.

Results: There was a significant overall effect of study condition $(P<0.001)$, with post hoc analyses revealing that mean daily step counts reported during the first week of the diary condition (9898 \pm 3002 steps/day) were significantly higher than those reported during the covert condition (8331 \pm 3010 steps/day) and during the second week of the diary condition (8226 \pm 3170 steps/day) $(P<0.001)$.

Conclusion: Reactivity to wearing unsealed pedometers and step count recording appears to last for 1 week. In the absence of any intervention material, step counts return to normal levels during the second week of monitoring, and therefore represent a more accurate estimate of habitual activity. These findings have important implications to both researchers and practitioners interested in the use of pedometers for physical activity surveillance and promotion. 
Keywords: habitual physical activity, objective assessment, self-monitoring, feedback, covert monitoring, physical activity promotion. 


\section{INTRODUCTION}

Paragraph number 1 Pedometers have become acceptable research tools for the objective assessment of ambulatory physical activity in different populations (23). They provide an inexpensive, accurate and reliable, objective measure of walking-based activities by counting the number of steps taken per day, enabling the accumulative measurement of occupational, leisure time and household activity, along with activity required for everyday transportation (12). Pedometers are widely used as both a surveillance tool for physical activity monitoring, and as a motivational device in physical activity interventions. The ability of the individual to receive immediate feedback on their accumulated step count is an important feature of the motivational aspect of the pedometer $(15,18)$. In intervention research, pedometer use has been associated with significant increases in physical activity (by approximately 2500 steps/day), and reductions in body weight, BMI, and blood pressure (3). The greatest changes in physical activity have been observed in those interventions which have incorporated step count recording and goal setting $(3,18)$.

Paragraph number 2 When used as a measurement tool, researchers often provide participants with unsealed pedometers (no restriction on participants viewing their step count) and request that they record their daily step count in an activity diary/step log. Concerns have been raised however on the impact of the feedback obtained from the pedometer on participant's activity levels (14). If activity changes as a result of wearing the pedometer, defined as reactivity (25), this could affect the validity of pedometer-determined activity data in surveillance studies. 
Paragraph number 3 To date there have been conflicting findings on the presence of reactivity to pedometers in adults. Behrens and Dinger (2) reported no evidence of reactivity occurring in response to wearing an accelerometer and an unsealed pedometer in young adults. Similarly Matevey et al.(15), Eastep et al.(11) and Marshall (14) have all reported minimal differences between unsealed and sealed (when the visible display of the pedometer is restricted) step counts in adult samples. Similar findings have been reported when pedometer reactivity has been assessed in children $(17,19,24)$.

Paragraph number 4 In the research outlined above $(2,14,15,17,19,24)$ participants have been aware in the sealed condition that they were wearing a pedometer, or a device that measures physical activity (11), which in itself may elicit some degree of reactivity. Only when participants are unaware that their activity levels are being monitored (termed covert monitoring) can a true investigation into reactivity be undertaken (1). Using covert monitoring, Clemes et al.(5) and Clemes and Parker (7) have shown a reactive effect occurring in response to wearing a pedometer and recording daily step counts in adults. For example, Clemes and Parker (7) examined the presence of reactivity to pedometers in response to wearing sealed and unsealed pedometers, with and without step count recording in the unsealed conditions. It was observed in this study that the greatest degree of reactivity occurred in response to recording daily step counts in a diary. In the sealed and unsealed (without step count recording) conditions, a small reactive response was observed over the first 2-3 days of monitoring, characterised by an increase in step counts occurring on these days. However within both of these conditions participants step counts returned to the levels observed in the covert condition on days 4 to 7 . In the diary condition, unlike the two other conditions, participants step counts remained elevated 
throughout the 7 day condition and it was suggested that reactivity to pedometers and step count recording appears to last for at least one week, however due to the short term nature of this condition, the exact duration of pedometer reactivity is unknown (7).

Paragraph number 5 Whilst in their study, Eastep et al.(11) observed little differences between step counts recorded using sealed and unsealed pedometers, they did observe that walking behaviour decreased in a linear fashion throughout their two 3-week study conditions, suggesting that some degree of reactivity was present in both their sealed and unsealed conditions. It was concluded from this research that a pedometer "feedback effect" may be present, but it may only last between one and two weeks (11). The aim of the current study therefore was to increase our knowledge of pedometer reactivity in free-living adults by examining the presence and duration of reactivity in response to wearing a pedometer and recording daily step counts in a diary.

\section{METHODS}

\section{Participants}

Paragraph number 6 A convenience sample of 90 adult participants (69\% female, age = $26.8 \pm 13.0$ years, BMI $=23.4 \pm 4.0 \mathrm{~kg} / \mathrm{m}^{2}$ ), blinded to the study aim, volunteered to participate. Participants were recruited from the staff and student population at Loughborough University, and were enrolled into the study after responding to recruitment material that asked for 'volunteers to take part in a study that examined the use of a new body posture monitor'. A health screen completed prior to enrolment into the study confirmed that participants were all in good general health and none had any physical illnesses or disabilities that might affect their normal daily routine. The study received ethical approval from the Loughborough University 
Ethical Advisory Committee, and participants provided written informed consent. At the study outset, body mass $(\mathrm{kg})$ and height $(\mathrm{cm})$ were directly measured without shoes using electronic weighing scales (Tanita UK Ltd) and a wall-mounted stadiometer (Seca UK). BMI was calculated as $\mathrm{kg} / \mathrm{m}^{2}$.

\section{Pedometer}

Paragraph number 7 The New Lifestyles NL-800 pedometer (New Lifestyles, Inc., Lees Summit, MO) was used in this study. This pedometer uses the same piezo-electric mechanism as the NL-2000 and NL-1000 pedometers which have been shown to accurately detect steps taken in both laboratory $(6,9,10,16,21)$ and free-living conditions $(6,20)$. The NL pedometer range all have internal memory chips and are capable of storing up to 7 days of data, in 1-day epochs. Their internal clock resets the step count at midnight.

\section{Procedure}

Paragraph number 8 During the first visit to the laboratory, participants were issued with a sealed pedometer and informed that the device was a 'new body posture monitor that measures time spent in horizontal, seated and standing postures' (covert condition), replicating an established protocol adopted in previous studies employing covert monitoring to examine pedometer reactivity $(5,7)$. The tape used to seal the pedometer would tear if removed, therefore non-compliance would be obvious when participants returned to the laboratory. Participants were shown the correct position to wear the device, on the midline of the thigh, and were requested to wear it throughout waking hours for seven days, only removing when either bathing, showering or swimming. During this condition participants were issued with a daily diary and 
asked to report the time the device was put on in the morning and taken off at the end of the day, along with any times and duration that the device was removed throughout the day.

Paragraph number 9 During the second visit to the laboratory, daily step counts measured from the past seven days were recorded into an activity log by an experimenter using the NL-800's memory function. Participants were informed that the device was actually a pedometer and they were invited to participate in a further condition which involved them wearing the pedometer unsealed, whilst continuing with their normal daily routine, for a period of 2 weeks. The duration of this condition was based on the suggestion that pedometer reactivity may last between one and two weeks (11). During this condition participants were issued with a second diary and requested to record their daily step counts each night upon going to bed (diary condition). Like the covert condition, participants also recorded the time the pedometer was put on and taken off each day, along with any times and durations that the pedometer was removed throughout the day. To control for any confounding effects of the day of the week (7), individual participants began the covert and diary conditions on the same day of the week. Upon completion of the 2-week diary condition participants returned the pedometer and diary to the laboratory. During this visit step counts recorded in the diary were verified against step counts stored in the pedometer's internal memory from the previous 7 days. 100\% agreement was observed for all participants between the diary entries and step counts recorded by the pedometer.

\section{Statistical Analyses}

Paragraph number 10 Statistical analyses were conducted using Predictive Analytics SoftWare (PASW, previously SPSS) for Windows version 18. Mean daily step counts and pedometer wear 
times (calculated in minutes per day) recorded during the covert condition and during weeks 1 and 2 of the diary condition were calculated and tested for normality using the one sample Kolmogorov-Smirnov test, which confirmed that the data were normally distributed. Mean daily step counts recorded in each condition were compared between BMI groups (participants were categorised as either normal weight [BMI $<25 \mathrm{~kg} / \mathrm{m}^{2}$ ] or overweight/obese [BMI $\left.\geq 25 \mathrm{~kg} / \mathrm{m}^{2}\right]$ ), males and females, and staff and students at the outset using one-way ANOVA's. Participants' mean daily step counts from each condition (covert, diary week 1 and diary week 2) were compared using a repeated-measures ANOVA, with sex, BMI group and employment status (staff/student) as between-subjects factors. Bonferroni adjusted pairwise comparisons were performed in the event of an overall significant effect of condition. Within each condition, to investigate any changes in step counts occurring over the study days (i.e. diary day 1, diary day 2 etc.), daily step counts recorded during each condition were compared using a repeated-measures ANOVA, with sex, BMI group and employment status (staff/student) as between-subjects factors. In the event of a significant study day-order effect Bonferroni corrected post hoc comparisons were undertaken. Statistical significance was set at $P<0.05$.

\section{RESULTS}

\section{Participants}

Paragraph number 11 Following the covert condition there was no evidence of any tampering with the pedometer seal, confirming that all participants were blinded to the fact that they were wearing a pedometer in this condition. All participants complied with the protocol and were included in the analyses. A series of one-way ANOVA's revealed that mean daily step counts recorded during the covert condition and during weeks 1 and 2 of the diary condition did not 
differ significantly between staff $(n=40)$ and students $(n=50)$, males $(n=28)$ and females $(n=$ 62), or participants classified as normal weight $(\mathrm{n}=61)$ and overweight/obese $(\mathrm{n}=29)($ all $P>$ 0.05). Due to the lack of differences observed between these groups, data are reported for the sample as a whole throughout.

\section{Pedometer wear time}

Paragraph number 12 Reported pedometer wear times during the covert condition and weeks 1 and 2 of the diary condition are shown in Table 1. Pedometer wear times did not vary significantly across the three study weeks $(\mathrm{F}=0.24, P=0.79)$.

\section{Overall effects of condition}

Paragraph number 13 Mean step counts recorded during the covert condition, and during weeks 1 and 2 of the diary condition are shown in Table 1 . There was a statistically significant difference between mean step counts recorded during each week (covert condition, diary condition week 1 and diary condition week 2$)$ of the study ( $\mathrm{F}=21.7, P<0.001)$. Bonferronicorrected post hoc analyses revealed that mean step counts reported during week 1 of the diary condition were significantly higher than those recorded during the covert condition ( $\mathrm{t}=6.8, P<$ $0.001)$ and during week 2 of the diary condition $(t=5.6, P<0.001)$. There were no significant interaction effects of sex, BMI group or employment status (staff/student) indicating that all participants responded similarly to the study conditions.

Insert Table 1 about here 


\section{The influence of study day order in each condition}

Paragraph number 14 There were no significant overall effects of study day order in the covert condition $(\mathrm{F}=1.0, P=0.42)$ or in week 2 of the diary condition $(\mathrm{F}=2.0, P=0.07)$ (Figure 1). A significant main effect of study day order was present during week 1 of the diary condition (F $=3.3, P=0.003)$, with Bonferroni-corrected post hoc analyses revealing that step counts reported on day 1 were significantly higher than step counts reported on days 4,5 and 7 ( $P<$ 0.002) (Figure 1). Within each week there were no significant interaction effects of sex, BMI group or employment status.

\section{Insert Figure 1 about here}

\section{DISCUSSION}

Paragraph number 15 This study aimed to increase our understanding of reactivity to pedometers in adults by examining the presence and duration of reactivity to wearing a pedometer and recording daily step counts in a diary. The study extends earlier work utilising covert monitoring to examine the true presence of reactivity in an adult sample $(5,7)$. As observed previously $(5,7)$, reactivity to wearing the pedometer and recording daily step counts did appear to exist during the first week of the diary condition, with step counts increasing significantly by 1567 (SD 2184) steps/day during this week in comparison to the covert condition. Mean daily step counts reported during the second week of the diary condition were significantly lower than those reported during the first week of this condition (step counts decreased by 1672 [SD 2815] steps/day) suggesting that in the sample studied, the reactivity response lasted for one week only. Indeed, there were no significant differences between mean 
step counts recorded during the covert condition and the second week of the diary condition (8331 versus 8226 steps/day). No significant interaction effects of sex, BMI group (normal weight/overweight including obese) or employment status (staff/students) were evident in the present study indicating that all participants responded similarly to the study conditions.

Paragraph number 16 The majority of studies examining the presence of reactivity to wearing pedometers and recording daily step counts in adults have been restricted to seven day monitoring periods $(5,7,15)$. For example, Clemes and Parker (7) observed that in their 7-day diary condition, step counts remained elevated (relative to the covert condition) throughout this period which led to the suggestion that reactivity appears to last for at least one week. A similar pattern in daily step counts was observed in the present study during the first week of the diary condition. Using a longer monitoring period, Eastep et al. (11) examined the effect of feedback from pedometers on walking behaviour in a sample of 21 adult participants enrolled in a 'Walking for Fitness' class. This study involved one group of participants wearing a sealed pedometer for three weeks followed by an unsealed pedometer for another three weeks. The order of conditions was reversed in a second group of participants. While no differences in mean step counts were observed between the two study conditions (sealed and unsealed), Eastep et al. (11) did observe that walking behaviour decreased in a linear fashion throughout the two 3-week conditions, suggesting that some degree of reactivity was present in both. It was concluded from this research that a pedometer "feedback effect" may be present, but it may only last between one and two weeks (11). The present findings support this suggestion by demonstrating that reactivity appears to be present during the first week of monitoring, but this effect diminishes during the second week. 
Paragraph number 17 A recent study in children, which used a 3-week monitoring period, observed a reactivity effect occurring in response to wearing sealed pedometers (13). Ling et al. (13) observed that step counts recorded during the first week of monitoring, in a sample of 133 children (aged 9-12 years), were significantly higher than step counts recorded during the third week of monitoring. It was concluded from this study that reactivity to sealed pedometers does exist in children and this should be taken into account in future studies interested in the assessment of habitual activity in this age group. A number of short term (one week duration) studies conducted in children and adults investigating the presence of reactivity to pedometers by either comparing sealed and unsealed step counts (without covert monitoring), or by comparing step counts across the individual days of monitoring, have concluded that reactivity does not appear to exist $(8,15,17,19)$. In contrast, evidence from studies employing longer monitoring periods of up to three weeks suggest that reactivity does appear to be present $(11,13)$, agreeing with the findings from short-term studies employing covert monitoring $(5,7)$. The findings from the present study suggest that reactivity lasts for the first week of monitoring and it is recommended that future studies investigating the presence of reactivity, without covert monitoring, adopt monitoring periods of at least two weeks to enable differences between the first and second weeks to be examined.

Paragraph number 18 A limitation of earlier studies examining pedometer reactivity is a lack of information on pedometer wear time $(5,7)$. It could be argued that in earlier work adopting covert monitoring when participants are aware that the device they are wearing is a pedometer, they simply wear it for a longer duration which results in more steps being counted. Wear time 
data was collected throughout the covert and diary conditions in the present study and it was found that pedometer wear time did not differ significantly between the study conditions. This finding demonstrates that the increases in step counts observed during the first week of the diary condition are not a function of wear time, and represent a true effect of reactivity to this condition.

Paragraph number 19 The implications of the findings of the present study are twofold. Firstly, when using unsealed pedometers with step count recording for the assessment of habitual physical activity in surveillance studies, or as a baseline measure in interventions, it is recommended that a 2-week monitoring period is adopted with the first week used as a familiarisation week only. Data collected during the second week will provide a more accurate reflection of usual or habitual activity levels. The second implication relates to the use of pedometers as a motivational tool. The present findings suggest that in pedometer intervention studies, simply issuing a pedometer to participants along with a daily diary will not be sufficient to lead to changes in physical activity levels after the first week. It is recommended that additional intervention strategies are adopted, such as encouraging participants to set weekly step count goals, and/or frequent contact between the participants and experimenter, in order to get the best out of the motivational characteristics of pedometers.

Paragraph number 20 The present study is not without its limitations. Whilst the sample comprised a relatively large group of both working aged adults and younger adults, the sample was not balanced in terms of males and females, or BMI groups. Only 6 (7\%) participants were classified as obese (BMI $\geq 30 \mathrm{~kg} / \mathrm{m}^{2}$ ), therefore it was not possible to investigate whether these 
individuals responded differently to the pedometer when compared to normal weight and overweight individuals. Participants were all recruited from one location in the UK and on average their step counts recorded during the covert condition (and week 2 of the diary condition) suggested that the sample were classified as 'somewhat active' (22). However, as participants responded to advertisements for volunteers to take part in a study 'that examined the use of a new body posture monitor", it is unlikely that the sample were biased towards those with an interest in physical activity. Indeed, step counts observed in the present study are similar to those observed in a larger, four-week pedometer surveillance study conducted in UK adults (4). It is recommended however that the findings of this study are confirmed in other populations, such as children and adults from other countries.

Paragraph number 21 In conclusion, the present findings suggest that reactivity to unsealed pedometers with step count recording lasts for a period of 1 week. It is recommended therefore that when unsealed pedometers and step count dairies are used for the assessment of habitual activity, researchers use a 2-week monitoring period, with the first week classified as a familiarisation week only. Step counts recorded during the second week will provide a more accurate reflection of habitual activity. When used as a motivational tool in free-living participants, it appears that in the absence of any further intervention material, the motivational characteristics of pedometers only last during the first week of use. The findings from the current study increase our understanding of pedometer reactivity in adults, and have important implications to both researchers and practitioners interested in the use of pedometers for physical activity surveillance and promotion. 


\section{ACKNOWLEDGMENTS}

The authors would like to acknowledge Silvia Costa for her help with data collection during this study.

\section{CONFLICTS OF INTEREST}

There are no perceived conflicts of interest associated with this research. The results of the present study do not constitute endorsement by ACSM.

\section{REFERENCES}

1. Beets M. The pursuit of the reactivity: A need for a closer look. Measurement in physical education and exercise science. 2006;10(4):265 - 67.

2. Behrens TK, Dinger MK. Motion sensor reactivity in physically active young adults. Res Q Exerc Sport. 2007;78(2):1-8.

3. Bravata DM, Smith-Spangler C, Sundaram V et al. Using pedometers to increase physical activity and improve health: a systematic review. JAMA. 2007;298(19):2296304.

4. Clemes SA, Hamilton SL, Lindley MR. Four-week pedometer-determined activity patterns in normal-weight, overweight and obese adults. Prev Med. 2008;46:325-30.

5. Clemes SA, Matchett N, Wane SL. Reactivity: an issue for short-term pedometer studies? Br J Sports Med. 2008;42(1):68-70.

6. Clemes SA, O'Connell S, Rogan LM, Griffiths PL. Evaluation of a commercially available pedometer used to promote physical activity as part of a national programme. Br J Sports Med. 2010;44(16):1178-83. 
7. Clemes SA, Parker RA. Increasing our understanding of reactivity to pedometers in adults. Med Sci Sports Exerc. 2009;41(3):674-80.

8. Craig CL, Tudor-Locke C, Cragg S, Cameron C. Process and treatment of pedometer data collection for youth: the Canadian Physical Activity Levels among Youth study. Med Sci Sports Exerc. 2010;42(3):430-5.

9. Crouter SE, Schneider PL, Bassett DR, Jr. Spring-levered versus piezo-electric pedometer accuracy in overweight and obese adults. Med Sci Sports Exerc. 2005;37(10):1673-9.

10. Crouter SE, Schneider PL, Karabulut M, Bassett DR, Jr. Validity of 10 electronic pedometers for measuring steps, distance, and energy cost. Med Sci Sports Exerc. 2003;35(8):1455-60.

11. Eastep E, Beveridge S, Eisenman P, Ransdell L, Shultz B. Does augmented feedback from pedometers increase adults' walking behavior? Percept Mot Skills. 2004;99(2):392402.

12. Hornbuckle LM, Bassett DR, Jr., Thompson DL. Pedometer-determined walking and body composition variables in African-American women. Med Sci Sports Exerc. 2005;37(6):1069-74.

13. Ling FC, Masters RS, McManus AM. Rehearsal and pedometer reactivity in children. $J$ Clin Psychol. 2011;67(3):261-6.

14. Marshall AL. Should All Steps Count When Using a Pedometer as a Measure of Physical Activity in Older Adults? J Phys Act Health. 2007;4(3):305-14. 
15. Matevey C, Rogers L, Dawson E, Tudor-Locke C. Lack of reactivity during pedometer self-monitoring in adults. Measurement in physical education and exercise science. 2006;10(1):1-11.

16. McClain JJ, Hart TL, Getz RS, Tudor-Locke C. Convergent validity of 3 low cost motion sensors with the ActiGraph accelerometer. J Phys Act Health. 2010;7(5):662-70.

17. Ozdoba R, Corbin C, Le Masurier G. Does reactivity exist in children when measuring activity levels with unsealed pedometers. Pediatr Exerc Sci. 2004;16:158-66.

18. Rooney B, Smalley K, Larson J, Havens S. Is knowing enough? Increasing physical activity by wearing a pedometer. WMJ. 2003;102(4):31-6.

19. Rowe D, Mahar M, Raedeke T, Lore J. Measuring physical activity in children with pedometers: Reliability, reactivity, and replacement of missing data. Pediatr Exerc Sci. 2004;16(343-354).

20. Schneider PL, Crouter SE, Bassett DR. Pedometer measures of free-living physical activity: comparison of 13 models. Med Sci Sports Exerc. 2004;36(2):331-5.

21. Schneider PL, Crouter SE, Lukajic O, Bassett DR, Jr. Accuracy and reliability of 10 pedometers for measuring steps over a 400-m walk. Med Sci Sports Exerc. 2003;35(10):1779-84.

22. Tudor-Locke C, Bassett DR, Jr. How many steps/day are enough? Preliminary pedometer indices for public health. Sports Med. 2004;34(1):1-8.

23. Tudor-Locke C, Bassett DR, Shipe MF, McClain JJ. Pedometry methods for assessing free-living adults. J Phys Act Health. 2011;8(3):445-53.

24. Vincent SD, Pangrazi RP. Does reactivity exist in children when measuring activity levels with pedometers? Pediatr Exerc Sci. 2002;14:56-63. 
25. Welk GJ, Corbin CB, Dale D. Measurement issues in the assessment of physical activity in children. Res Q Exerc Sport. 2000;71(2 Suppl):S59-73. 
Table 1. Self-reported pedometer wear times and mean daily step counts recorded during each week/condition of the study for the sample as a whole $(n=90)$. Data are presented as the mean and SD.

\begin{tabular}{lcc}
\hline & $\begin{array}{c}\text { Wear time } \\
(\text { mins })\end{array}$ & Steps/day \\
& $820(128)$ & $8331(3010)$ \\
\hline Covert & & \\
Diary week 1 & $810(116)$ & $9898(3002)^{*}$ \\
Diary week 2 & $811(137)$ & $8226(3170)$
\end{tabular}

* Mean daily step counts recorded during week 1 of the diary condition were significantly higher than those recorded during the covert condition and week 2 of the diary condition. 


\section{Figure legend}

Figure 1. Mean daily step counts recorded during each study day within each condition, along with standard error bars. To control for any confounding effects of the day of the week, individual participants began the covert and diary conditions on the same day of the week. For each participant therefore day 1 of each study week falls on the same week day.

\section{Figure 1}

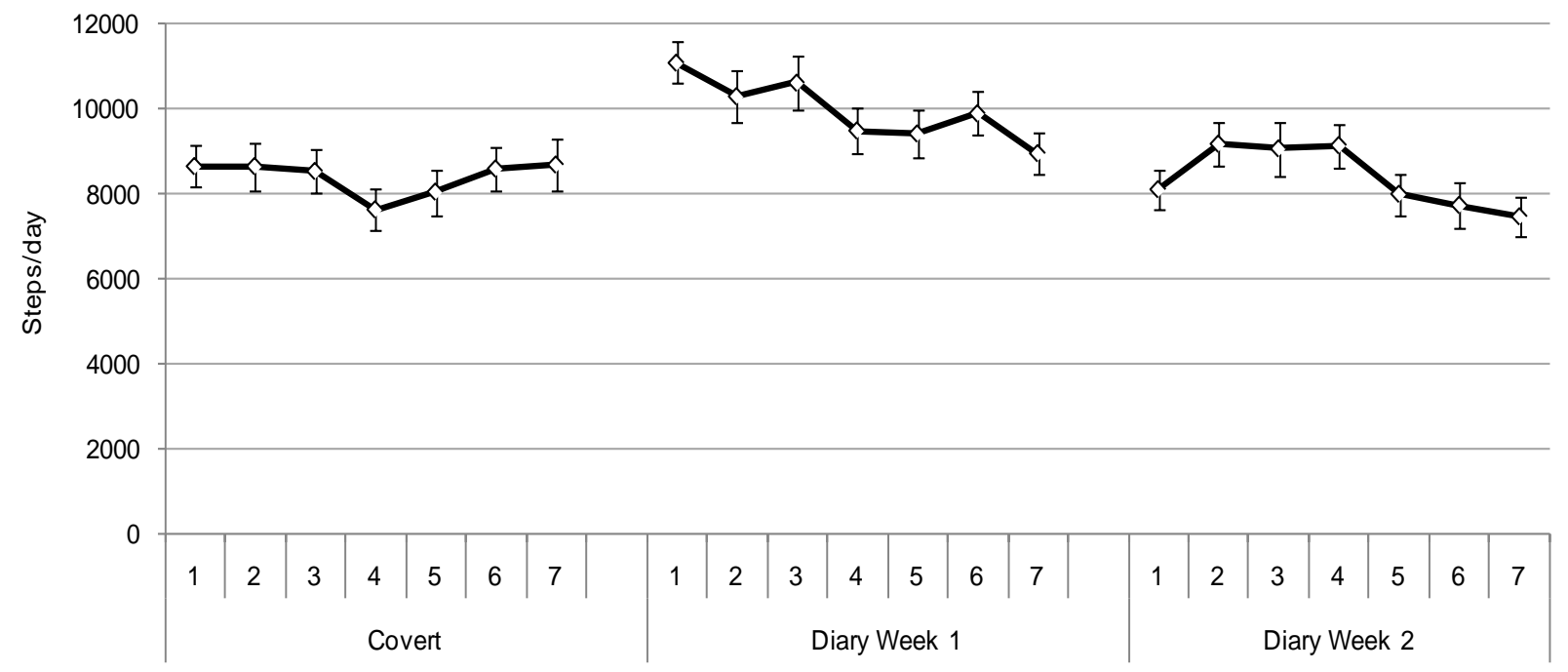

Study condition 